\title{
THE
}

UNIVERSITY

University of Rhode Island

OF RHODE ISLAND

DigitalCommons@URI

Physics Faculty Publications

Physics

7-16-1990

\section{Quantum Images of Hamiltonian Chaos}

Niraj Srivastava

Gerhard Müller

University of Rhode Island, gmuller@uri.edu

Follow this and additional works at: https://digitalcommons.uri.edu/phys_facpubs

Terms of Use

All rights reserved under copyright.

\section{Citation/Publisher Attribution}

N. Srivastava and G. Müller. Quantum images of Hamiltonian chaos. Physics Letters A 147 (1990), 282-286.

Available at: http://www.sciencedirect.com/science/article/pii/037596019090448W

This Article is brought to you for free and open access by the Physics at DigitalCommons@URI. It has been accepted for inclusion in Physics Faculty Publications by an authorized administrator of DigitalCommons@URI. For more information, please contact digitalcommons-group@uri.edu. 


\title{
Quantum images of Hamiltonian chaos
}

\author{
Niraj Srivastava and Gerhard Müller \\ ${ }^{1}$ Department of Physics, University of Rhode Island, Kingston RI 02881-0817, USA
}

\begin{abstract}
A novel way of demonstrating and visualizing quantum manifestations of Hamiltonian chaos is presented. The method is based on the calculation of invariants in the form of time averages of dynamical variables in microscopic states. The observed quantum nonintegrability effects are unmistakable images of well-understood phenomena of classical Hamiltonian chaos.
\end{abstract}

The curious phenomenon of deterministic randomness is without question the hallmark of chaos. In nonintegrable classical Hamiltonian systems, chaotic phase-space trajectories are ubiquitous. They are informationally incompressible and, therefore, (intrinsically or effectively) deterministically random. Redundancy is either absent (homoplectic situation) or irretrievable (autoplectic situation) $[1,2]$. However, the paradigmatic significance of chaos in physical systems is truly warranted only if that phenomenon is understandable in the context of quantum mechanics and describable in quantum mechanical terms [3]. The still unresolved issue of the existence and nature of quantum chaos is therefore of fundamental importance. Many quantum nonintegrability effects have been identified in recent years and are currently being studied in great detail [4]. However, the comparison between quantum manifestations of nonintegrability and classical manifestations of Hamiltonian chaos has been rather indirect for the most part. The goal of this communication is to report, for a particular dynamical system, a new direct link between well-understood phenomena of classical Hamiltonian chaos and some striking quantum nonintegrability effects.

Consider a system of two localized quantum spins specified by an interaction Hamiltonian of the form

$$
\hat{H}=\hbar^{2} \sum_{\mu=x y z}\left\{-J_{\mu} \hat{\sigma}_{1}^{\mu} \hat{\sigma}_{2}^{\mu}+\frac{1}{2} A_{\mu}\left[\left(\hat{\sigma}_{1}^{\mu}\right)^{2}+\left(\hat{\sigma}_{2}^{\mu}\right)^{2}\right]\right\} .
$$

It defines, in the limit $\hbar \rightarrow 0, \sigma \rightarrow \infty, \hbar \sqrt{\sigma(\sigma+1)}=s, \hbar \hat{\sigma}_{l}^{\mu}=S_{l}^{\mu}$, an autonomous classical Hamiltonian system $H\left(\mathbf{S}_{1}, \mathbf{S}_{2}\right)$ of two degrees of freedom for a pair of three-component vectors of length $s$,

$$
\mathbf{S}_{l}=s\left(\sin \vartheta_{l} \cos \varphi_{l}, \sin \vartheta_{l} \sin \varphi_{l}, \cos \vartheta_{l}\right), \quad l=1,2 .
$$

The classical time evolution is specified by the equations of motion $d \mathbf{S}_{l} / d t=-\mathbf{S}_{l} \times \partial H / \partial \mathbf{S}_{l}$. A set of canonical coordinates is given by $p_{l}=s \cos \vartheta_{l}, q_{l}=\varphi_{l}$. The classical two-spin system is completely integrable if there exists an independent integral of the motion in addition to $H$. The integrability condition is nontrivial,

$$
\left(A_{x}-A_{y}\right)\left(A_{y}-A_{z}\right)\left(A_{z}-A_{x}\right)+J_{x}^{2}\left(A_{y}-A_{z}\right)+J_{y}^{2}\left(A_{z}-A_{x}\right)+J_{z}^{2}\left(A_{x}-A_{y}\right)=0,
$$

and a second integral of the motion (analytic invariant) $I\left(\mathbf{S}_{1}, \mathbf{S}_{2}\right)$ is explicitly known for the integrable cases [5]. In this Letter we shall present results for the one-parameter model $H_{\alpha}$ specified by interaction constants $J_{x}=J_{y}=1, J_{z}=0,-A_{x}=A_{y}=\alpha, A_{z}=0$, which is nonintegrable except for $\alpha=0, \pm 1$. Different nonintegrable spin models have been used previously for quantum chaos studies [6].

Integrability implies that the flow in four-dimensional (4d) phase space is confined to $2 \mathrm{~d}$ invariant tori. Each torus is specified by the values of two action variables $J_{1}, J_{2}$, and the two independent analytic invariants are expressible (at least in principle) as $H\left(\mathbf{S}_{1}, \mathbf{S}_{2}\right)=E\left(J_{1}, J_{2}\right)$ and $I\left(\mathbf{S}_{1}, \mathbf{S}_{2}\right)=I\left(J_{1}, J_{2}\right)$. However, invariants can be reconstructed numerically via time averages for 
quite general (integrable and nonintegrable) situations. The method of reconstruction, which is designed for classical invariants, is readily translated into quantum mechanics. We shall demonstrate that some of the most dramatic nonintegrability effects in Hamiltonian systems can be observed in the properties of such invariants, both classically and quantum mechanically.

For the classical system, pick any dynamical variable $A$ which is independent of $H$ and determine its time average over the phase-space trajectory specified by the initial condition $\left(\mathbf{S}_{1}, \mathbf{S}_{2}\right)$ :

$$
\langle A\rangle=\lim _{T \rightarrow \infty} \frac{1}{T} \int_{0}^{T} d t A\left(T ; \mathbf{S}_{1}, \mathbf{S}_{2}\right) .
$$

According to the Birkhoff theorem [7], this invariant is defined almost everywhere in phase space. Its properties depend sensitively on whether the phase flow is integrable or not [8]. In integrable cases of the classical two-spin model, the entire phase space is densely foliated by invariant tori: rational tori (dense, measure zero) and irrational tori (dense, measure one). For initial conditions on irrational tori, the invariant (3) is a piecewise smooth function of two variables (rather than four initial conditions); these two variables are the action coordinates $J_{1}, J_{2}$ which specify each torus. Suppose we determine two independent invariants $\left\langle M_{x}^{2}\right\rangle$ and $\left\langle M_{z}^{2}\right\rangle$ in this way, $M_{\mu}=\left(S_{1}^{\mu}+S_{2}^{\mu}\right) / 2$, then all points with coordinates $\left(\left\langle M_{x}^{2}\right\rangle^{1 / 2},\left\langle M_{z}^{2}\right\rangle^{1 / 2}, E\right)$ fall on a piecewise smooth surface. Every point on that invariant-surface is the image of a point on the action plane $\left(J_{1}, J_{2}\right)$.

For nonintegrable cases of the two-spin model, the phase flow is qualitatively different. It consists of intact irrational tori, which are nowhere dense anymore, and chaotic trajectories, which are dense everywhere. These two types of trajectories exhaust the measure of phase space. Periodic orbits and invariant Cantor sets, which are also present in phase space (and important in different contexts), have zero measure. Predictably, the chaotic phase flow has a dramatic effect on the invariants $\left\langle M_{x}^{2}\right\rangle^{1 / 2}$ and $\left\langle M_{z}^{2}\right\rangle^{1 / 2}$. What was a piecewise smooth surface in $\left(\left\langle M_{x}^{2}\right\rangle^{1 / 2},\left\langle M_{z}^{2}\right\rangle^{1 / 2}, E\right)$-space now has partially disintegrated.

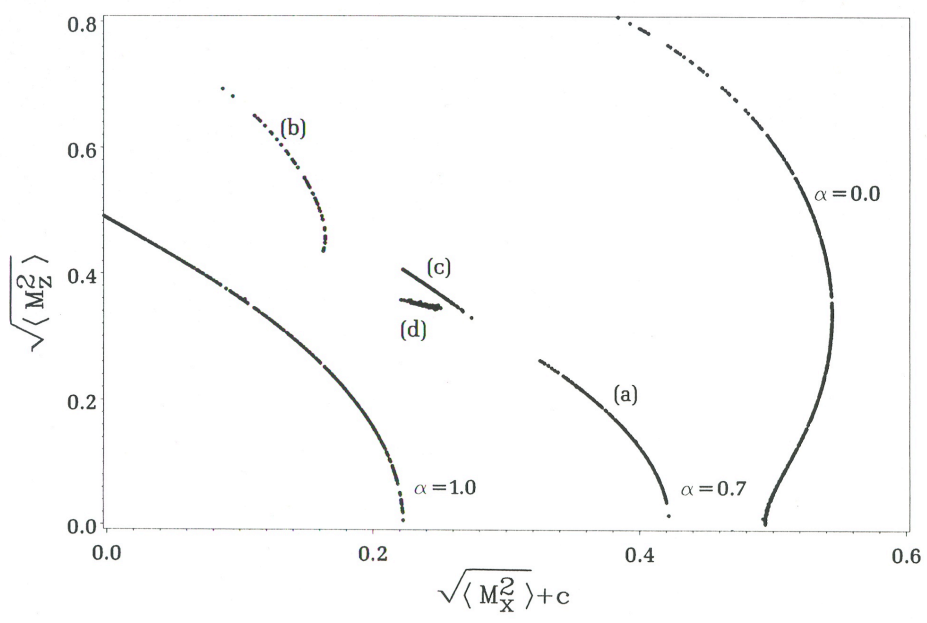

Figure 1. Invariant $\left\langle M_{x}^{2}\right\rangle^{1 / 2}$ versus invariant $\left\langle M_{z}^{2}\right\rangle^{1 / 2}$ at energy $E=0.2$ for the cases $\alpha=0$, $\alpha=0.7$ and $\alpha=1$ of the classical two-spin model $H_{\alpha}$ with $s=1$. For better display, the sections for $\alpha=0$ and $\alpha=1$ have been shifted horizontally by the amounts $c=0.2$ and $c=-0.2$, respectively. The invariants have been determined by time averages over individual trajectories for initial conditions randomly chosen on the three energy surfaces. The number of initial conditions chosen was 500 for $\alpha=0.7$ and 600 for $\alpha=0$ and $\alpha=1$. The results of 5 time averages were deleted because of extremely poor convergence.

For a demonstration of the invariant-surface or its remnants, consider the one-parameter model $H_{\alpha}$. Fig. 1 shows the section at $E=0.2$ of the invariant-surface $\left(\left\langle M_{x}^{2}\right\rangle^{1 / 2},\left\langle M_{z}^{2}\right\rangle^{1 / 2}, E\right)$ for the integrable cases $\alpha=0$ and $\alpha=1$, and of its remnants for the non integrable case $\alpha=0.7$. We have 
determined these invariants via time average (3) over individual trajectories for initial conditions randomly chosen on the energy surface [8]. For the nonintegrable case $\alpha=0.7$, we show in fig. 2 the Poincaré surface of section $\left(\vartheta=\pi / 2, \vartheta_{2}<0\right)$ for a number of different trajectories at $E=0.2$. The letters (a-c) relate three different types of invariant tori in fig. 2 to three distinct parts of the invariant-surface shown in fig. 1. The cluster of points labelled (d) in fig. 1 originate from initial conditions in the region of widespread chaos in fig. 2. In the integrable limit $\alpha=0$, the entire surface of section is densely foliated by type (b) tori, giving rise to a perfectly smooth invariant-surface as shown in fig. 1. With $\alpha$ increasing from zero, type (a) tori make their appearance, separated from type (b) tori by a separatrix in the form of a chaotic band of increasing width. Furthermore, type (c) tori emerge from within the chaotic band and become quite prominent at $\alpha=0.7$. Upon further increase of $\alpha$, the chaotic band starts to shrink again and disappears in the integrable limit $\alpha=1$ along with the type (b) and type (c) tori, leaving a dense foliation of typ (a) tori, resulting again in a perfectly smooth invariant-surface (see Fig. 1).

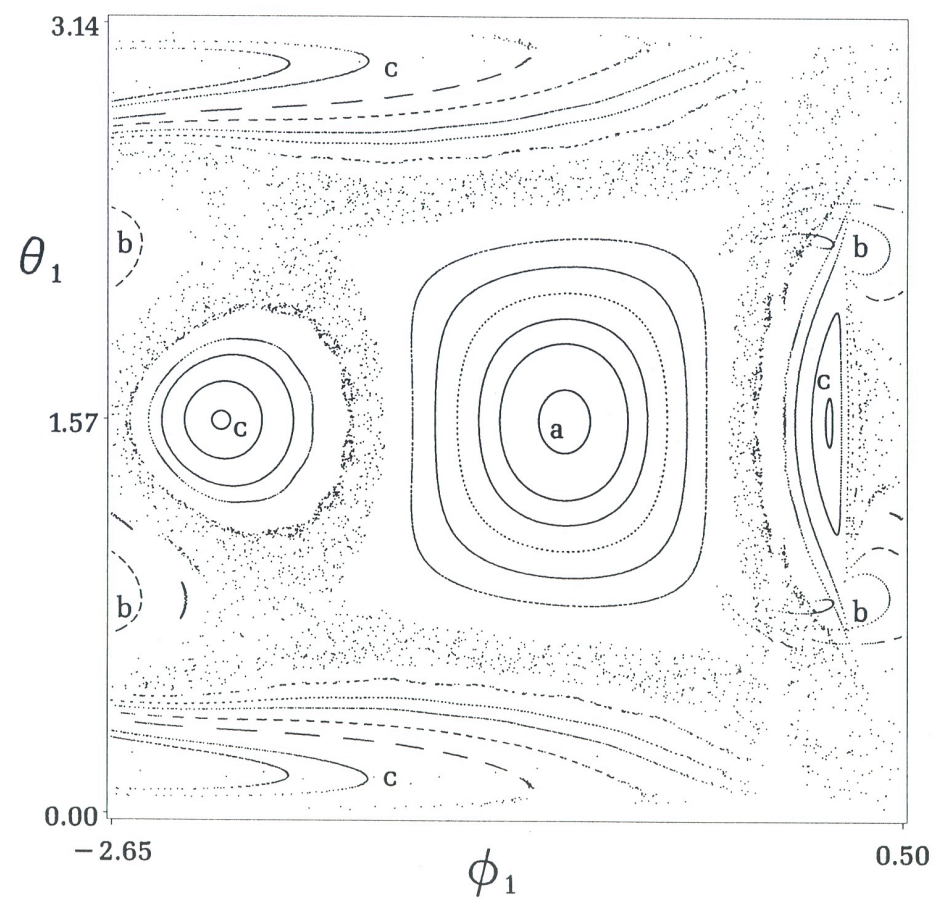

Figure 2. Poincaré surface of section $\left(\vartheta=\pi / 2, \dot{\vartheta}_{2}<0\right)$ projected onto the $\left.\vartheta_{1}, \phi_{1}\right)$-plane of a number of phase-space trajectories at energy $E=0.2$ for the nonintegrable classical two-spin model $H_{\alpha=0.7}$ with $s=1$.

For the nonintegrable case $\alpha=0.7$ shown in fig. 1 , we observe that parts of the invariantsurfaces corresponding to the integrable limits $\alpha=0$ and $\alpha=1$ are still visibly present at the top and bottom, respectively. These parts originate from phase-space regions in which, respectively, type (b) tori and type (a) tori are predominant. However, because of the omnipresence of chaos in phase space, the invariant-surface is no longer perfectly smooth anywhere. One interruption occurs on a scale large enough to be visible in fig. 1 with all its characteristic features. For initial conditions within the widespread chaotic region, the points $\left(\left\langle M_{x}^{2}\right\rangle^{1 / 2},\left\langle M_{z}^{2}\right\rangle^{1 / 2}\right)$ tend to cluster at (d) near the center of the gap between the two remnants of the invariant-surface. If it were not for the slow convergence of the time averages along chaotic trajectories due to low flux cantori $[8,9]$, the entire chaotic region would be represented by a single isolated point in the constant-energy section. In the full $\left(\left\langle M_{x}^{2}\right\rangle^{1 / 2},\left\langle M_{z}^{2}\right\rangle^{1 / 2}, E\right)$-space, these points form string-like objects, at least on a coarse-grained scale. Also in the gap between the two residual parts of invariant-surface, some of the time averages 
$\left\langle M_{x}^{2}\right\rangle^{1 / 2},\left\langle M_{z}^{2}\right\rangle^{1 / 2}$ form a new piece of invariant-surface which originates from initial conditions on secondary type (c) tori. This piece of invariant-surface has no smooth counterpart in anyone of the integrable limits. The characteristic pattern of invariant-surface interrupted by gaps and the gaps populated with invariant-strings and new pieces of invariant-surface repeats itself on smaller scales ad infinitum.

We shall now demonstrate how these striking nonintegrability effects identified in invariants for the classical two-spin cluster make their appearance in the invariants of the corresponding quantum two-spin cluster. The numerical reconstruction of invariants via time averages can be translated into quantum mechanics according to a scheme previously explored by Peres [10]: take any operator $\hat{A}$ with $[\hat{A}, \hat{H}] \neq 0$, thus representing a dynamical variable which is independent of $\hat{H}$, and consider the matrix elements of $\hat{A}(t)$ in the energy representation,

$$
\left\langle\lambda|\hat{A}(t)| \lambda^{\prime}\right\rangle=\left\langle\lambda \hat{A} \mid \lambda^{\prime}\right\rangle \exp \left(i\left(E_{\lambda}-E_{\lambda^{\prime}}\right) t / \hbar\right) .
$$

Performing the time average wipes out all off-diagonal elements

$$
\overline{\left\langle\lambda|\hat{A}(t)| \lambda^{\prime}\right\rangle} \equiv \lim _{T \rightarrow \infty} \frac{1}{T} \int_{0}^{T} d t\left\langle\lambda|\hat{A}(t)| \lambda^{\prime}\right\rangle=\langle\hat{A}\rangle_{\lambda} \delta_{\lambda \lambda^{\prime}}
$$

and thus defines the quantum invariant $\langle\hat{A}\rangle$, which is diagonal in the energy representation as it should be. This construction can be carried out for any $(2 \sigma+1)$-dimensional representation of operators, i.e. for any (integrable or nonintegrable) spin- $\sigma$ case of the general quantum spin Hamiltonian (1). However, the properties of $\langle\hat{A}\rangle$ depend sensitively on whether the classical integrability condition (2) is satisfied or not. In the case where degenerate energy levels occur, the eigenvectors in the invariant subspaces must be chosen such that all off-diagonal matrix elements $\left\langle\lambda \hat{A} \mid \lambda^{\prime}\right\rangle$ are zero.

For integrable cases of our two-spin system (1), the quantum energy spectrum $E_{\lambda}$ is naturally catalogued as a two-parameter family in terms of two quantum numbers $m_{1}, m_{2}$, each representing one of two action variables $J_{k}=m_{k} \hbar, m_{k}=-\sigma,-\sigma+1, \ldots, \sigma, k=1,2$, in accordance with semiclassical quantization. However, we do not need to know the function $E\left(J_{1}, J_{2}\right)$ to recover this order inherent in the quantum energy spectrum. Suppose we determine the same two invariants $\left\langle\hat{M}_{x}^{2}\right\rangle$ and $\left\langle\hat{M}_{z}^{2}\right\rangle$ for $\hat{M}_{\mu}=\hbar\left(\hat{\sigma}_{1}^{\mu}+\hat{\sigma}_{2}^{\mu}\right) / 2$ quantum mechanically as we did classically and represent each eigenstate $|\lambda\rangle$ as a point with coordinates $\left\langle\hat{M}_{x}^{2}\right\rangle_{\lambda}^{1 / 2},\left\langle\hat{M}_{z}^{2}\right\rangle_{\lambda}^{1 / 2}, E_{\lambda}$ in $3 \mathrm{~d}$ space. Then the set of points representing any particular symmetry class of eigenstates of $\hat{H}$ can be connected to a fully intact $2 \mathrm{~d}$ quantum invariant-web with four bonds per vertex. Locally, the web resembles a square lattice with spacing of $\mathrm{O}(\hbar)$. This invariant-web is the direct quantum counterpart of the piecewise smooth classical invariant-surface $E\left(\left\langle M_{x}^{2}\right\rangle^{1 / 2},\left\langle M_{z}^{2}\right\rangle^{1 / 2}\right)$ discussed earlier. Each vertex of the web represents a quantized torus, claiming an area $\Delta \Omega \simeq \hbar^{2}$, the size of one mesh, in accordance with the uncertainty principle.

For nonintegrable cases of the two-spin model (specifically the case $\alpha=0.7$ of $\hat{H}_{\alpha}$ ), we thus expect that the quantum invariant-web is affected by the presence of chaos in a way which is in direct relation to our observations made in fig. 1 for the classical invariant-surface. Fig. 3 shows a portion of the invariant-web for this particular case projected onto the $\left(\left\langle\hat{M}_{z}^{2}\right\rangle_{\lambda}^{1 / 2}, E_{\lambda}\right)$-plane. Shown are all eigenstates of $\hat{H}_{\alpha=0.7}$ for $\sigma=45$ (within a window of given size) which transform according to the irreducible representations A1A (full circles) and B1S (open squares) of the symmetry group $D_{2} \otimes S_{2}$, where $S_{2}$ is the permutation group and $D_{2}$ contains all twofold rotations about the coordinate axes [11]. The arrow in fig. 3 indicates the energy at which the classical invariant-surface is intersected for presentation in fig. 1.

The correspondence between classical and quantum non integrability effects in this representation is indeed quite remarkable. In the regular regions at top left and bottom right, the invariantweb is fully intact; here classical chaos is confined to areas much smaller than the mesh size. Between the two regular regions extends a broad band of chaos along a separatrix of the classical motion. Here the invariant-web is interrupted, which signals the destruction of quantized tori. The presence of a separatrix is indicated by the observation that one set of lines changes from positive 


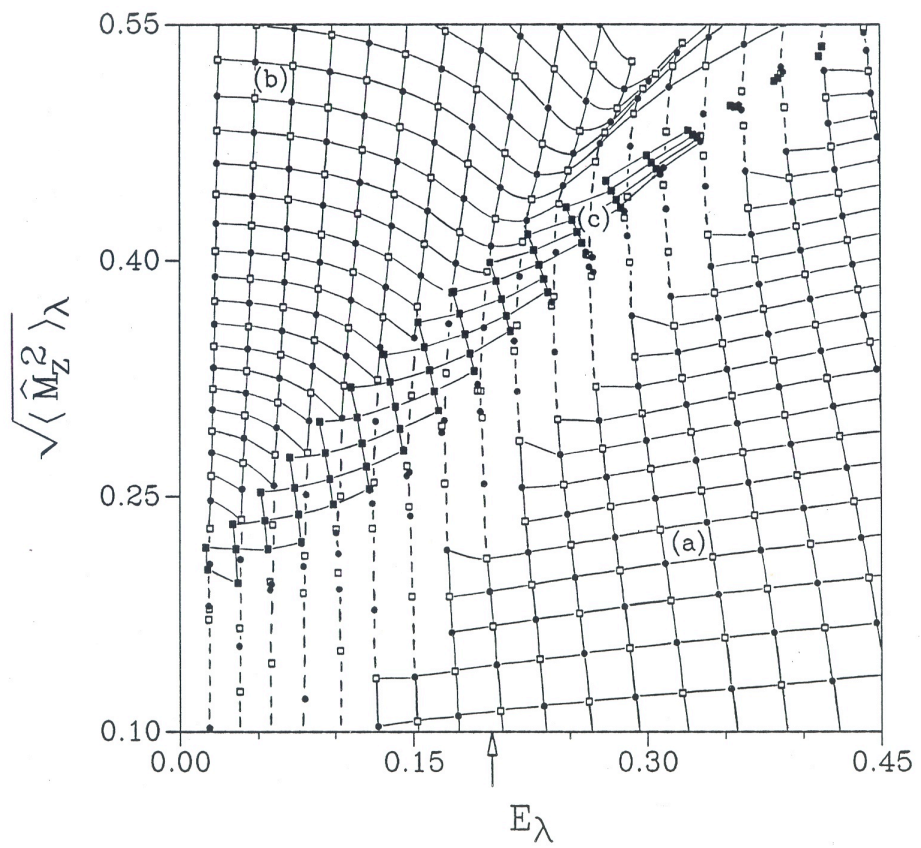

Figure 3. Invariant $\left\langle\hat{M}_{z}^{2}\right\rangle^{1 / 2}$ versus energy eigenvalue $E_{\lambda}>0$ for an eigenstates of symmetry classes A1A (full circles) and B1S (open squares) of the nonintegrable quantum two-spin model $\hat{H}_{\alpha=0.7}$ with spin quantum number $\sigma=45$ and $s=1$. Only states within a certain window of values for $\left\langle\hat{M}_{z}^{2}\right\rangle^{1 / 2}$ and $E_{\lambda}$ are shown.

slope to negative slope going through a point of infinite slope if interpolated smoothly across the chaotic region (dashed lines). Since these lines can be interpreted as lines of constant action, say $J_{2}=$ const, the conclusion is that one of the two fundamental frequencies of the classical time evolution, $\omega_{1}=\partial E / \partial J_{1}$, slows down to zero, thus marking the location of a separatrix in the action plane.

Within the chaotic region, the quantum states tend to cluster in short strips along the dashed lines. In that region, these lines approximate lines of constant (and invariant) energy. This clustering tendency of chaotic states leaves regions bordering the intact invariant-web depleted of states; we have observed the same effect in the classical invariant-surface shown in fig. 1. Note that the clustering of chaotic states is accompanied by a slight displacement perpendicular to the lines of constant energy. The amount of level repulsion appears to be insignificant in this representation, but if all states of that symmetry class are projected onto the energy axis, the mean level spacing has the same order of magnitude. Superimposed onto the region populated by the chaotic states (in the projection of fig. 3), we find a new web of quantized type (c) tori, a web which is disconnected from the primary web and which has its classical counterpart in fig. 1 as discussed before. Note also that in the projection of fig. 3, the A1A and B1S states avoid each other in regions (a) and (b), whereas they lie on top of one another in region (c).

In summary, the classical and quantum invariants as represented in this study, provide a novel direct link between manifestations of Hamiltonian chaos and quantum nonintegrability effects. The structure of the Hamiltonian phase flow has its precise image in the classical invariant-surface and an image of finite resolution in the quantum invariant-web. The destruction of invariant tori is paralleled by the disappearance of quantum numbers and the restoration of secondary KAM tori by the reappearance of new quantum numbers. A more detailed account of the wealth of information which can be gleaned from such comparative studies of invariants in nonintegrable quantum and classical dynamical systems of few degrees of freedom will be published in due course. 
We thank Charles Kaufman for useful comments and suggestions. This work was supported in part by the US National Science Foundation, Grant DMR-86-03036 and by Sigma Xi, the Scientific Research Society. The numerical calculations were performed on the CRAY-2 of the National Center for Supercomputing Applications, University of Illinois at Urbana-Champaign. We have used a modified cmpj.sty style file.

\section{References}

1. S. Wolfram, Phys. Rev. Lett. 55 (1985) 449.

2. J. Ford, Phys. Today 33, no. 4 (1983) 40.

3. J. Ford, in: Directions in chaos, Vol. I, ed. H. Bai-Lin (World Scientific, Singapore, 1988).

4. B. Eckhardt, Phys. Rep. 163 (1988) 205, and references therein.

5. E. Magyari, H. Thomas, R. Weber, C. Kaufman and G. Müller, Z. Phys. B 65 (1987) 363. 6 K. Nakamura and A.R. Bishop, Phys. Rev. B 33 (1986) 1963; M. Feingold, N. Moiseyev and A. Peres, Phys. Rev. A 30 (1984) 509; H. Frahm and H.J. Mikeska, Z. Phys. B 60 (1985) 117.

6. J.E. Marsden and R. Abraham, Foundations of mechanics, 2nd Ed. (Benjamin/Cummings, Reading, MA, 1985) p. 238.

7. N. Srivastava, C. Kaufman, G. Müller, R. Weber and H. Thomas, Z. Phys, B 70 ( 1988) 251.

8. J.D. Meiss and E. Ott, Physica D 20 (1986) 387.

9. A. Peres, Phys. Rev, Lett. 53 (1984) 1711.

10. P.W. Atkins, M.S. Child and C.S.G. Phillips, Tables for group theory (Oxford Univ. Press, Oxford, 1970) 